\title{
Application of Structural Principles to the Design of Triptycene-Based Molecular Gears with Parallel Axes
}

\author{
Derik K. Frantz ${ }^{\S}$ Kim K. Baldridge, and Jay S. Siegel ${ }^{\star}$ \\ §SCS Poster Prize Winner
}

\begin{abstract}
This perspective discusses the important aspects of the design of molecular gear systems based on triptycene. Molecular systems are categorized into three classes according to the orientation of the gear axes: i) bent (bevel gears), ii) linear, and ii) parallel (spur gears). To date, no examples of molecular gears in which the axes of rotation lie in parallel have been reported; correspondingly, the bulk of this report focuses on the factors one must evaluate to construct such systems.
\end{abstract}

Keywords: Correlated rotation · Dynamic gearing · Molecular gears · Triptycene

\section{Introduction}

Artificial molecular rotors, ${ }^{[1]}$ particularly those with potential applications in the field of molecular devices ${ }^{[2]}$ and machines, ${ }^{[3]}$ represent contemporary chemical miniaturizations of macroscopic engineering constructs. Among the most ubiquitous components in macroscopic machinery for the past two millennia has been the gear, ${ }^{[4]}$ which transforms rotational motion from one axis to another or into translational motion. Substituted triptycene propellers (Tp, 1 (Fig. 1)), the most common rotators found in molecular gear systems, were first shown by Ōki and coworkers ${ }^{[5]}$ to contain

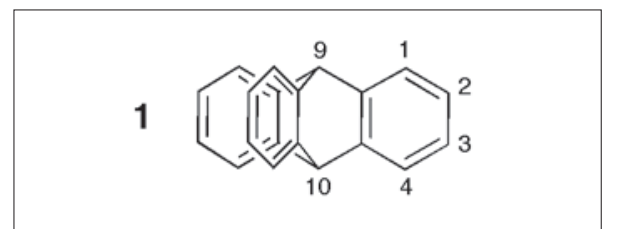

Fig. 1.
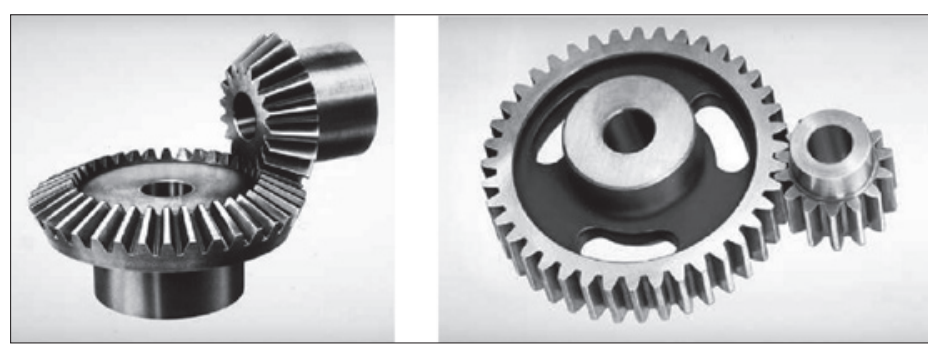

Fig. 2. Macroscopic bevel (left) and spur (right) gears. Photographs courtesy of Emerson Power Transmission Corporation (www. emerson-ept.com) sterically well-defined blades, as several 9-substituted derivatives of Tp have high energetic barriers to rotation due to the meshing of the gear-like parts. In principle, Tp units can adopt parallel, bent, or linear orientations, the first two mimicking macroscopic spur and bevel gears respectively (Fig. 2). ${ }^{[6]}$

These findings led the groups of Mislow ${ }^{[7]}$ and Iwamura ${ }^{[8]}$ independently to utilize Tp-based systems to develop the first molecular systems exhibiting dynamic gearing, ${ }^{[9]}$ defined as the "special effect on the rate or mechanism of a process that may be attributed to the intermeshing of a chemical rotor with a neighboring group". [10]

These and all other molecular gears that have been synthesized (i.e. the rotators have been held at angles considerably higher than $0^{\circ}$ ) evoke the image of molecular analogs to macroscopic bevel gears. To date, no example of molecular spur gears - systems in which the gears have parallel axes of rotation - have been reported. ${ }^{[11]}$

\footnotetext{
${ }^{*}$ Correspondence: Prof. Dr. J. S. Siegel

University of Zürich

Institute of Organic Chemistry

Winterthurerstrasse 190

$\mathrm{CH}-8057$ Zürich

Tel.: +41446354281

Fax: +4144635 6888

E-mail: jss@oci.uzh.ch
}

\section{Triptycene, Interacting Linear Rotators, and Beveled Gearing}

A molecule of $\mathrm{Tp}$ resembles a threeblade gear. The molecule has overall $D_{3 h}$ symmetry and contains a three-fold axis of rotation that intersects the 9- and 10-positions (Fig. 3). ${ }^{[12]}$ The height of each blade is $7.2 \AA$, measured as the distance between the edges of the van der Waals radii of $\mathrm{H}-\mathrm{C}(1)$ and $\mathrm{H}-\mathrm{C}(4)$. The length of each blade, measured from the axis of rotation to the edge of the van der Waals radius of $\mathrm{H}-\mathrm{C}(2)$ and $\mathrm{H}-\mathrm{C}(3)$, is $5.9 \AA$. As a consequence of the three-fold axis of rotation, the blades are separated by $120^{\circ}$ angles. The rotational profile of triptycene roughly resembles a cylinder, and the largest distance from the circular edge of this cylinder to the intersection of the van der Waals surfaces of the two blades is $3.8 \AA .{ }^{[13]}$

When two rotating triptycene moieties are positioned in such a way that their cylindrical gearing profiles overlap, steric interactions will be present. Before analyzing the situations in which dynamic gearing occurs, let us consider a few examples where the angle between interacting rotators is $180^{\circ}$. The rotational barrier about the 9,9' $\mathrm{C}-\mathrm{C}$ bond in 9,9'-bitriptycyl[14] (2, Fig. 4) was found to be in excess of 54

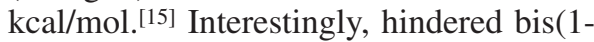
methyltriptycene-9-yl)ethyne (3) was the first published compound in which rotational hindrance about a $\mathrm{C}-\mathrm{C}$ triple bond was observed.[16] 


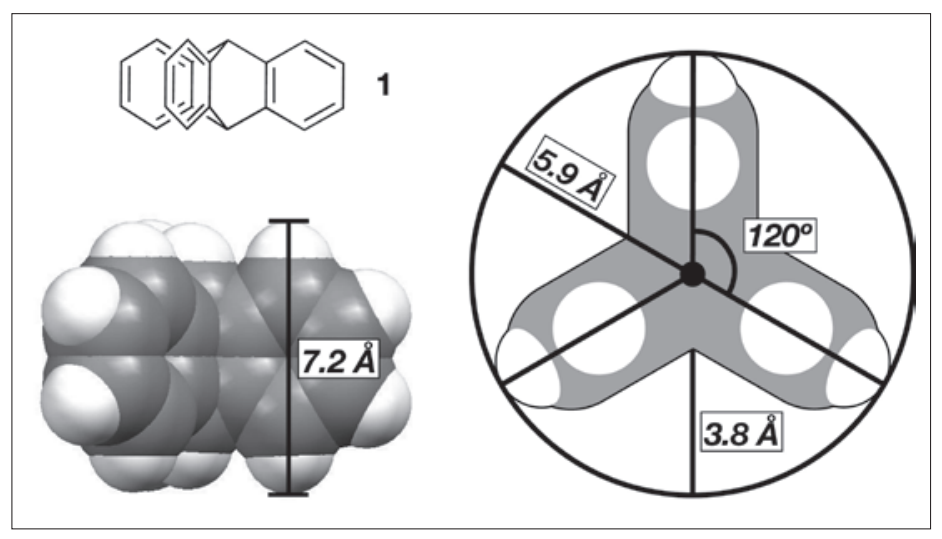

Fig. 3. Geometrical properties of Tp: (left) molecular structure of $\mathrm{Tp}$ as viewed perpendicular to the three-fold axis; (right) scaled drawing of triptycene (with hydrogen atoms in white) and circular rotational profile.

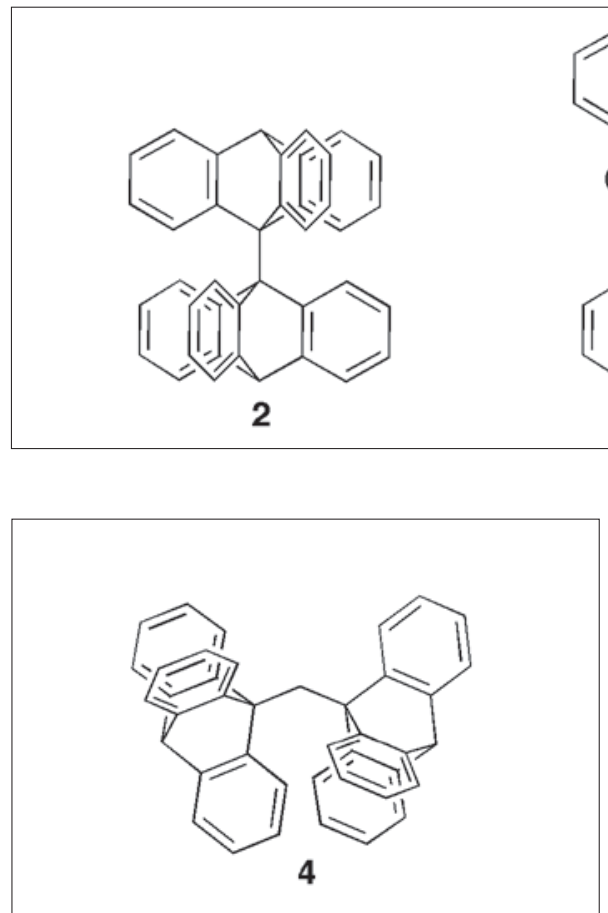

Fig. 5.

Rotational properties of other derivatives of bis(triptycen-9-yl)ethyne have been studied extensively by Toyota, Ōki, and coworkers. ${ }^{[17]}$ Other examples in which two rotators interact while being held at an angle of $180^{\circ}$ are hexasubstituted benzenetricarbonylchromium complexes, ${ }^{[18]}$ where steric bulk hinders rotation about the Ar$\mathrm{Cr}$ coordinate covalent bond. The linear relationship between the rotators allows for debate concerning the relationship between the rotators' rotation. One could argue that disrotation is conserved; if observed from a point along the axis, between the two rotators, the rotators rotate in opposite directions. On the other hand, one could argue that rotation of the rotators becomes conrotatory at an interacting angle of $180^{\circ}$, as both rotators appear to rotate in the same direction if such a molecule is observed from a distance.

Systems such as $\mathbf{2}$ and $\mathbf{3}$ exhibit static, not dynamic, gearing. ${ }^{[9]}$ Dynamic gearing is achieved when rotation is correlated and, correspondingly, when the process through which gear slippage occurs requires higher energies than correlated rotation. To analyze beveled triptycene gears, consider (9-Tp) ${ }_{2} \mathrm{CH}_{2}$ (4, Fig 5), a representative example of the systems developed by Mislow and Iwamura. ${ }^{[9]}$

The molecular structure of $4^{[19]}$ provides insight into its function as a remarkably efficient molecular gear system (Fig. 6). The molecule is highly strained, with a bond angle $\mathrm{C}(9)_{\mathrm{Tp}}-\mathrm{CH}_{2}-\mathrm{C}\left(9^{\prime}\right)_{\mathrm{Tp}}$ equal to $129^{\circ}$. If angle vectors are extended to $\mathrm{C}(10)_{\mathrm{Tp}}$ and $\mathrm{C}\left(10^{\prime}\right)_{\mathrm{Tp}}$, the angle becomes even wider, to $138^{\circ}$. The distance from the carbon of the methylene group to the center of the propeller hub (the point at the center of the line connecting $\mathrm{C}(9)$ and $\mathrm{C}(10))$ is 2.9 $\AA .{ }^{20]}$ These geometrical constraints force $\mathrm{H}-\mathrm{C}(1)$ to fit snugly between two benzene rings of the adjacent Tp moiety.

Further analysis of $\mathbf{4}$ reveals steric interactions between the benzene rings on each Tp group that do not allow for gear slippage without significant bending of the blades. Gear slippage requires the bond angle centered at the carbon atom of the methylene group to open even further than in its already strained state. The lowest energy ground state of $\mathbf{4}$ is of $C_{2}$ symmetry. An approximate $C$ conformation of $\mathbf{4}$ corresponds to a transition state of geared rotation, and the transition state to gear slippage is of $C_{2}$, symmetry[21] (Fig. 7). The energy difference between the structures of $C_{2}$ and $C_{s}$ symmetry is calculated to be only $0.2 \mathrm{kcal} / \mathrm{mol},{ }^{[22]}$ indicating an extremely low energetic requirement for gearing. In contrast, gear slippage requires significantly more energy, as the $C_{2 v}$ structure lies $30 \mathrm{kcal} / \mathrm{mol}^{[23,24]}$ higher in energy than the $C_{2}$ conformation.

In an attempt to quantify the efficiency of molecular gears, we introduce two terms: gearing fidelity and gearing temperature. Gearing fidelity refers to the quotient of the rates governing gearing $\left(k_{\text {geared rotation }}\right.$ ' $\left.k_{\text {slippage }}\right)$ at a given temperature, standardized at room temperature $(298 \mathrm{~K})$. Gear fidelity represents the average number of geared rotations that takes place per gear slip. In the case of $\mathbf{4}$, standard gearing fidelity equals roughly $7 \cdot 10^{21}$, indicating that these rotators gear with exceptional efficiency. ${ }^{[25]}$ Gearing temperature is the temperature under which the rate of gear slippage $\left(k_{\text {slippage }}\right)$ is slower than $1 \mathrm{~s}^{-1}$. For $\mathbf{4}$, the gearing temperature is $538 \mathrm{~K}$. These terms taken together provide characterization of the transfer-of-momentum efficiencies of molecular gears and the temperatures at which gears slip (particularly useful for diastereomerization processes of desymmetrized systems ${ }^{[23]}$ ).

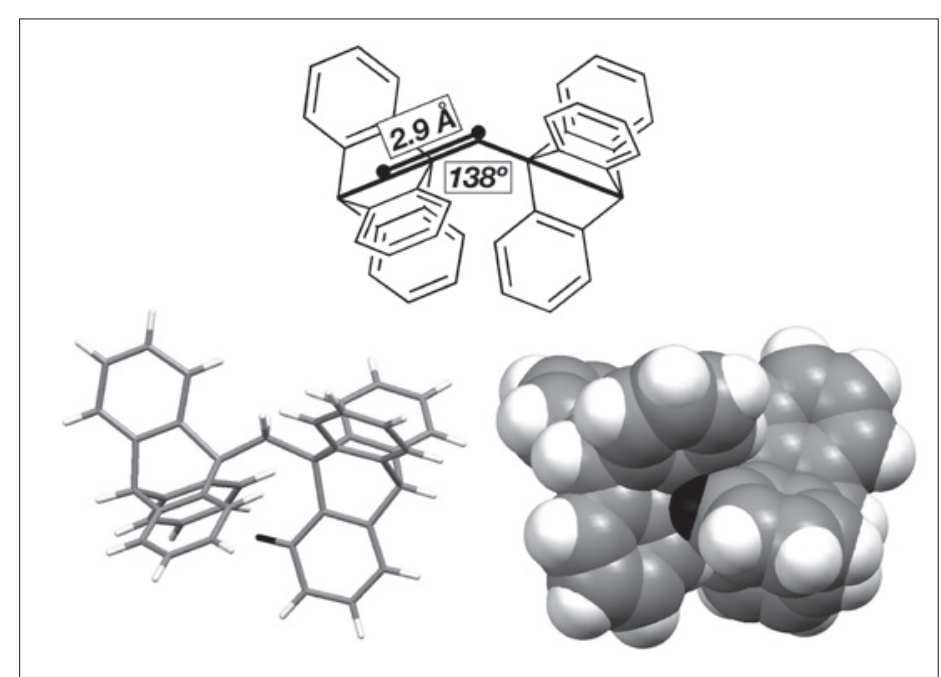

Fig. 6. (top)

Geometrical properties of 4. (bottom) Molecular structure of $\mathbf{4}$, rendered in capped stick (left) and spacefilling (right) models. The hydrogen atom that is intermeshed between the benzene rings of the neighboring Tp group is marked in black. 


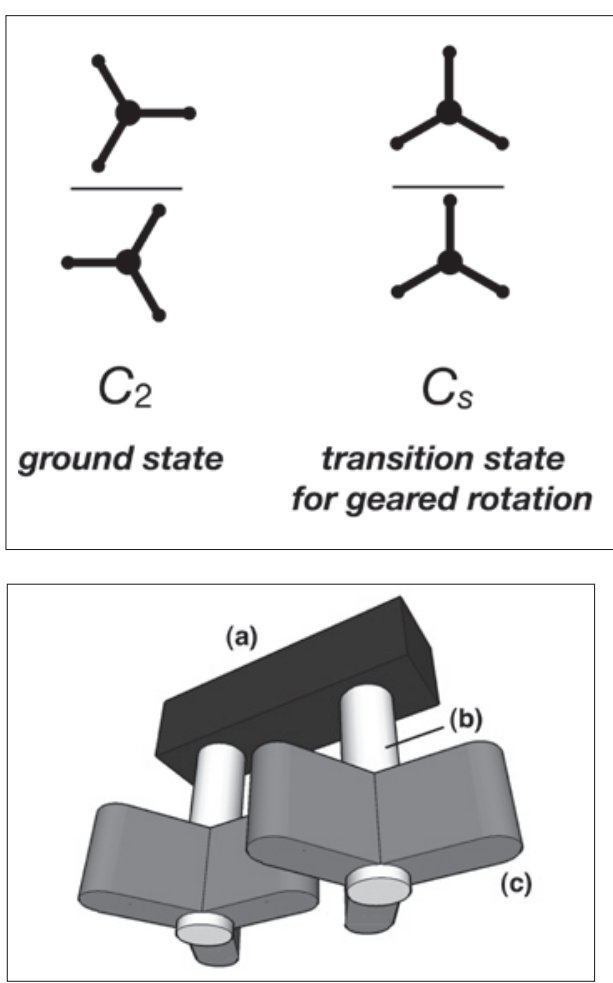

Fig. 8. Schematic representation of a molecular spur gear system with a common stator (a), axles (b), and rotators (c).

\section{From Bevel Gears to Spur Gears}

To design Tp-based molecular spur gears (Fig. 8), one must consider the differences between the interactions of Tp moieties that are separated by an angle of $\sim 130^{\circ}$ and those that lie in parallel. Unlike the case of 4 , where $\mathrm{H}-\mathrm{C}(1)$ wedges tightly between two benzene rings of the complementary Tp moiety, the entire edge of a benzene ring would have to fit into this cavity for gearing to take place in parallel Tp rotators. Accordingly, a major aspect in the design of a molecular spur gear is the distance between the two axes of rotation (Fig. 9). For gearing to occur, either the $C_{2}$ or the $C_{s}$ conformation must be the minimum energy structure, and the $C_{2 v}$ symmetry form the maximum energy conformation. Accordingly, if the conformation with $C_{2}$ symmetry is the ground state structure of lowest energy, the $C_{s}$ conformation must be the transition state to gearing and the $C_{2 v}$ structure must be the transition state for gear slippage. The minimum distance between the axes of rotation is $7.0 \AA$ in the $C_{2}$ conformation, $8.1 \AA$ in the $C_{s}$ conformation (transition state to gearing) and $7.2 \AA$ in the $C_{2 v}$ conformation (transition state to gear slippage). Gear slippage in 4 requires the cores of the Tp groups to move apart through significant stretching of the Tp-C-Tp angle. However, the opposite effect would take place when the Tp moieties lie in parallel, as geared rotation requires more separation of the core axes of the Tp groups than gear slippage. From this analysis, it can be concluded that steric
Fig. 7. The relative energies of these symmetric conformations determine the existence and efficiency of geared rotation.

\section{transition state for gear slippage}

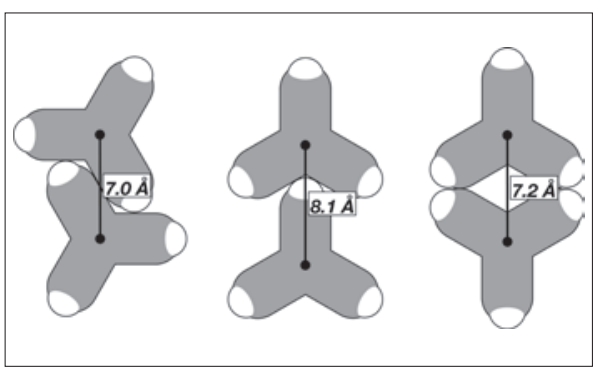

Fig. 9. Scaled, space-filling representations of Tp groups in parallel and minimum axis-axis distances in the $C_{2}$ (left), $C_{s}$ (center), and $C_{2 v}$ (right) conformations.

forces alone will not likely suffice to hinder gear slippage.

For gearing to take place between two Tp moieties that lie in parallel, the $C$ conformation must be lower in energy than the $C_{2 v}$ conformation. In the absence of a sterically induced hindrance to gear slippage, stabilization of the $C_{s}$ conformation and/or destabilization of the $C_{2 v}$ conformation must take place for gearing to occur with any fidelity. The $C_{2}$ conformation minimizes the space between the triptycene moieties and maximizes contact of the van der Waals surfaces. Additionally, attractive edge-to-face contacts are present and likely stabilize the system. ${ }^{[26]}$ Edge-to-face contacts are main- tained in the $C_{s}$ conformation, as $\mathrm{H}-\mathrm{C}(2)$ and $\mathrm{H}-\mathrm{C}(3)$ wedge between two benzene rings of the interacting Tp group. In contrast, the $C_{2 v}$ conformation reduces van der Waals contacts to a minimum and places four hydrogen atoms close together, likely introducing repulsive, destabilizing $\delta^{+}-\delta^{+}$interactions. Further destabilization of the $C_{2 v}$ conformation could arise from the presence of a vacuum between the four benzene rings. Although lack of strong steric interactions would certainly imply that gearing fidelities be significantly lower than what is observed in $\mathbf{4}$, the propensity to preserve van der Waals attractions may favor rotation though the $C$ conformation (the transition state for geared rotation) in Tp-based systems with parallel axes. It is important to note that extending the lengths of the blades will not significantly change the relative distances between the $C$ and $C_{2 v}$ conformations. Extending the blade length by $n$ extends the Tp-Tp distance of the $C_{s}$ and $C_{2 v}$ conformations by $n$ (in $C_{2 v}$ : $\left.2 n \cdot \cos \left(60^{\circ}\right)=n\right)$.

Assuming that correlated rotation can occur in Tp rotators that lie in parallel, designing such a system requires the selection of an appropriate combination of stator and axles. Suitable stators must be rigid and capable of projecting substituents in parallel. Several classes of compounds can accommodate these requirements, so we limit our discussion to derivatives of acenes, phenylenes, crescent molecules, and biindenes. The distance between the relevant positions that project in parallel is also of crucial importance to the design, as the Tp-Tp distance must be close enough to allow attractive van der Waals interactions to take place but far enough to allow the $C_{s}$ conformation to be reached without excessive strain.

The various length extents that can be obtained from acenes, crescents, phenylenes and biindene derivatives are shown (Fig. 10). As members of the graphene class of compounds, complementary acene (except

acenes

Fig. 10. Distances between indicated positions that project in parallel in potential stators based on acene, crescent, phenylene, and biindene derivatives. 
naphthalene) and crescent molecules have distances that are roughly identical. The distances in phenylenes are similar to those of the graphenes, with the exception of phenylene itself. Parallel positions in biindene derivatives lie at a distance of $\sim 8.3 \AA$, roughly the same distance necessary to achieve the $C$ conformation. Selection of appropriate axles is also critical to the system's ability to participate in correlated rotation. The axles must be relatively rigid and linear, and alkynes are the most obvious choice.

\section{Conclusion}

In this short perspective, triptycene and beveled molecular gearing were briefly discussed and considerations for the design of molecular spur gears were assessed. In our current research, we have begun to design and explore synthetic pathways to various Tp-scaffold constructs. In addition, we are applying computational methods to investigate mechanistic aspects of Tp-based molecular gears in which the axes are parallel.

January 15, 2009

[1] For an exceptional review of artificial molecular rotors, see G. S. Kottas, L. I. Clarke, D. Horinek, J. Michl, Chem. Rev. 2005, 105, 1281.

[2] a) A. P. de Silva, H. Q. N. Gunaratne, T. Gunnlaugsson, A. J. M. Huxley, C. P. McCoy, J. T. Rademacher, T. E. Rice, Chem. Rev. 1997, 97, 1515; b) T. W. Bell, N. M. Hext, Chem Soc. Rev. 2004, 33, 589

[3] a) E. R. Kay, D. A. Leigh, F. Zerbetto, Angew. Chem., Int. Ed. 2007, 46, 72; b) V. Balzani, A. Credi, F. M. Raymo, J. F. Stoddart, Angew. Chem., Int. Ed. 2000, 39, 3348.

[4] D. W. Dudley, 'The Evolution of the Gear Art', Dual Printing, Buffalo, 1969
[5] a) G. Yamamoto, M. Ōki, Chem. Lett. 1979, 1251; b) G. Yamamoto, M. Ōki, Chem Lett. 1979, 1255; c) G. Yamamoto, M. Ōki, Bull. Chem. Soc. Jpn. 1981, 54, 473: d) G. Yamamoto, M. Ōki, Bull. Chem. Soc. Jpn. 1981, 54, 481.

[6] The pictures in Fig. 2 were first brought to our attention by Kottas et al. in Ref. [1].

[7] a) W. D. Hounshell, C. A. Johnson, A. Guenzi, F. Cozzi, K. Mislow, Proc. Natl. Acad. Sci. 1980, 77, 6961; b) F. Cozzi, A. Guenzi, C. Johnson, K. Mislow, W. D. Hounshell, J. F. Blount, J. Am. Chem. Soc. 1981, 103, 957.

[8] a) Y. Kawada, H. Iwamura, J. Org. Chem. 1980, 45, 2547; b) Y. Kawada, H. Iwamura, J. Am. Chem. Soc. 1981, 103, 958.

[9] H. Iwamura, K. Mislow, Acc. Chem. Res. 1988, $21,175$.

[10] W. D. Hounshell, L. D. Iroff, D. J. Iverson, R. J. Wroczynski, K. Mislow, Isr. J. Chem. 1980 , 20,65 .

[11] One example of a bis(triptycyl) system in which the Tp groups lie close to parallel has been reported; however the stators are flexible and correlated rotation was not confirmed. J. C. Bryan, R. A. Sachleben, A. A. Gakh, G. J. Bunick, J. Chem. Crystallogr. 1999, 29, 513.

[12] For the molecular structure in Fig. 3, see A. S. Batsanov, T. B. Marder, Private Communication to the Cambridge Structural Database, identifier TRIPCN02, 2004.

[13] In Fig. 3 and 8, the drawings of Tp from the perspective of looking down on the axis are drawn to scale. The benzene rings have a thickness of $3.40 \AA$, the van der Waals diameter of a carbon atom. The hydrogen atom at the tip has a van der Waals radius of $1.20 \AA$. In Fig. 7, the minimum distance between axes were calculated from van der Waals contacts of inflexible Tp moieties.

[14] For the crystal structure of 4, see M. H. P. Ardebili, D. A. Dougherty, K. Mislow, L. H. Schwartz, J. G. White, J. Am. Chem. Soc. 1978, 100, 7994.

[15] L. H. Schwartz, C. Koukotas, C. Yu, J. Am. Chem. Soc. 1977, 99, 7710.

[16] P. Koo Tze Mew, F. Vögtle, Angew. Chem., Int. Ed. Engl. 1979, 18, 159.

[17] a) S. Toyota, T. Yamamori, M. Asakura, M. Ōki, Bull. Chem. Soc. Jpn. 2000, 73, 205; b) S. Toyota, T. Yamamori, T. Makino, M. Ōki, Bull. Chem. Soc. Jpn. 2000, 73, 2591; c) S. Toyota,
T. Yamamori, T. Makino, Tetrahedron 2001, 57, 3521.

[18] a) K. V. Kilway, J. S. Siegel, J. Am. Chem. Soc. 1992, 114, 255; b) P. A. Downton, B Mailvaganam, C. S. Frampton, B. G. Sayer, M. J. McGlinchey, J. Am. Chem. Soc. 1990, 112, 27.

[19] C. A. Johnson, A. Guenzi, R. B. Nachbar Jr., J. F. Blount, O. Wennerstroem, K. Mislow, J. Am. Chem. Soc. 1982, 104, 5163.

[20] This value assumes a $\mathrm{C}(9)_{\mathrm{Tp}}-\mathrm{CH}_{2}$ distance of $1.54 \AA$.

[21] It was calculated that the transition state to gear slippage actually involves $C_{\mathrm{s}}$ conformation that deviates slightly from $C$ symmetry. See H.-B. Bürgi, W. D. Hounshell, R. B. Nachbar Jr., K. Mislow, J. Am. Chem. Soc. 1983, 105, 1427.

[22] Refs. [6a] and [15].

[23] This value refers to the structure mentioned in Ref. [15].

[24] Experimental confirmation of barriers to gear slippage requires analysis of barriers of diastereomerization in phase isomers of desymmetrically labeled Tp rotators. This concept is explained, and data for many desymmetrized systems is supplied, in Ref [8], and references therein. The barrier of gear slippage cannot be calculated for $\mathbf{4}$ because the three propeller blades are equal by symmetry.

[25] Rate constants are calculated from $\Delta \mathrm{G}^{\neq}$values according to the Eyring equation. The Arrhenius equation can be used if pre-exponential factor $\mathrm{A}$ is known. For a listing of values of $\mathrm{A}$ in desymmetric derivatives of $\mathbf{4}$, see Ref. [8].

[26] F. Cozzi, J. S. Siegel, Pure \& Appl. Chem. 1995, $67,683$. 